\title{
Shine Bright Like a Diamond: Microfocus X-ray Sealed Tube Sources with Diamond Hybrid Anode Technology
}

\author{
J. Graf, ${ }^{1}$ T. Stuerzer, ${ }^{2}$ H. Ott, ${ }^{2}$ M. Mrosek, ${ }^{2}$ M. Benning, ${ }^{3}$ B. Noll, ${ }^{3}$ R. Durst, ${ }^{2}$ \\ P. Radcliffe, ${ }^{1}$ C. Michaelsen ${ }^{1}$ \\ ${ }^{1}$ Incoatec $\mathrm{GmbH}$, Geesthacht, Germany \\ ${ }^{2}$ Bruker AXS GmbH, Karlsruhe, Germany \\ ${ }^{3}$ Bruker AXS Inc., Madison (WI), USA
}

Diamond exhibits several unique properties, such as high thermal conductivity, low thermal expansion, extreme hardness, chemical inertness and a fairly high transmission coefficient for $\mathrm{X}$-ray radiation. The thermal conductivity of diamond is about 5 times higher than that of copper and the highest known conductivity of all bulk materials. Therefore, diamond, being a non-toxic material, is increasingly replacing traditional materials for the thermal management in challenging applications where a high local heat load needs to be dissipated, such as in heat sinks for highpower microelectronic devices.

The high transmission coefficient, even at low X-ray energies, fosters the use of diamond in X-ray components such as carrier membranes in X-ray lithography or $\mathrm{X}$-ray windows. In X-ray sources, diamond can be used as a transparent heat sink directly coupled to the anode material. This improves the heat dissipation considerably and allows for an increase in tube brilliance by applying a higher power load on the anode.

The I $\mu S$ DIAMOND is a new type of microfocus sealed tubes using a unique anode technology, the diamond hybrid anode. It takes advantage of the exceptional high thermal conductivity of diamond by using a bulk industrial diamond as a heat sink, which is coated with a thin layer of the target material, such as $\mathrm{Cu}$, Mo or Ag. The diamond heat sink makes the heat dissipation in a diamond hybrid anode more efficient. Consequently, the I IS DIAMOND can accept a higher power density in the focal spot on the anode without damaging the surface of the target layer. The balanced heat management in the source assures that the intensity loss over time is only a few percent over $10,000 \mathrm{~h}$ of full power operation, which is significantly lower than the intensity degradation observed for microfocus rotating anode sources. As a consequence, the intensity of the I $\mu$ S DIAMOND is about $20 \%$ higher than the average intensity output of a modern microfocus rotating anode.

The I $\mu S$ DIAMOND establishes a new class of X-ray sources, combining an intensity output that exceeds the average intensity of a microfocus rotating anode with all the comfort and lifetime of a standard microfocus sealed tube with a bulk anode. In this presentation, we will be discussing the main features of the I $\mu$ S DIAMOND and presenting selected results to demonstrate the impact of this new class of microfocus sealed tube X-ray sources on the data quality for applications, such as protein and small molecule crystallography. 